\title{
Neue ambulante Tarifstruktur TARDOC: eine Notwendigkeit
}

Mario Morgera, Patrick Müller

${ }^{a}$ Dr. oec., Leiter Tarife bei curafutura; ${ }^{b}$ Leiter Abteilung Ambulante Versorgung und Tarife der FMH

Die Tarifpartner curafutura und FMH haben im Juli 2019 die neue ambulante Tarifstruktur TARDOC beim Bundesrat eingereicht. Mit dem TARDOC liegt erstmals seit Jahrzehnten eine aktualisierte und sachgerechte Einzelleistungstarifstruktur vor. Nun liegt es am Bundesamt für Gesundheit BAG zu entscheiden, wie rasch der veraltete TARMED durch einen neuen und tarifpartnerschaftlichen Tarif abgelöst werden kann.

Der TARMED bildet aktuell die Tarifstruktur für die Abrechnung der ambulanten Leistungen ab und wurde per 1. Januar 2004 als national einheitliche Tarifstruktur für ambulante Leistungen eingeführt. Er umfasst gegenwärtig mehr als 4600 Positionen für sämtliche ärztlichen und wenige nicht ärztliche Leistungen in der Arztpraxis und im ambulanten Spitalbereich. Aufgrund von Blockaden in der dafür vorgesehenen Trägerorganisationen TARMED Suisse konnte der TARMED nie wirklich weiterentwickelt und gepflegt werden. Dadurch ist er veraltet, nicht mehr sachgerecht und widerspiegelt nicht mehr die betriebswirtschaftliche und medizinische Realität. Kein Wunder, ist der TARMED in den politischen Fokus geraten.

\section{Entwicklungsgeschichte des neuen Tarifs}

Die bisherige Geschichte der TARMED-Revision ist lange und dornenreich. Was im Jahr 2010 im Alleingang durch die FMH gestartet wurde, konnte Ende 2012 in einem Revisionsprojekt mit dem Verband der Unfallversicherer MTK und dem Spitalverband $\mathrm{H}+$ weitergeführt und schlussendlich im Jahr 2015 mit der Beteiligung von curafutura in ein gemeinsames ambulantes Tarifbüro überführt werden. Das Ziel all dieser Anstrengungen war es, eine komplett aktualisierte, neue und sachgerechte ambulante Einzelleistungstarifstruktur zu erarbeiten, um den TARMED abzulösen. Nach intensiven Verhandlungen in den letzten drei Jahren konnten die beiden Tarifpartner curafutura und FMH am 12. Juli 2019 die neue Tarifstruktur TARDOC beim Bundesrat einreichen (siehe auch den Artikel in der Ausgabe 2019;100[31-32]:1008-10). Damit zeigen curafutura und FMH, zusammen mit der MTK (welche den Tarif nicht genehmigen lassen muss), dass die Tarifpartnerschaft funktioniert, wenn die Beteiligten verhandlungs- und kompromissbereit sind. Zudem sind sich die beiden Tarifpartner einig, dass vorherrschende Probleme in der Tarifierung besser durch tarifpartnerschaftliche Expertise, Know-how und Einsatz gelöst werden, als durch Eingriffe durch Behörden oder durch Amtstarife. Eingriffe können immer nur partielle Korrekturen ermöglichen, die zudem aufgrund der Interdependenzen der Gesamtstruktur oftmals negative Auswirkungen auf andere Bereiche haben.

Das BAG wird nun im Auftrag des Bundesrates die eingereichte Tarifstruktur hinsichtlich der gesetzlichen Vorgaben prüfen und das weitere Vorgehen festlegen. Damit obliegt es nun dem EDI und dem BAG, zu entscheiden, wie rasch der veraltete TARMED durch einen neuen, aktualisierten und tarifpartnerschaftlichen Tarif abgelöst werden kann.

\section{Volkswirtschaftliche und gesellschaft- liche Bedeutung}

Der Stillstand der letzten 15 Jahre im TARMED hat zu einer gefährlichen Resignation im Schweizerischen Gesundheitswesen geführt. Obwohl der Reformbedarf immer deutlicher auf der Hand lag, schwand der Glauben an der effektiven Reformfähigkeit des Tarifs. Dabei handelt es sich wertmässig gesehen um den mit Abstand wichtigsten Tarif im Schweizer Gesundheitswesen: Über den ärztlichen ambulanten Tarif wurden 2018 rund 11 Mrd. Franken abgerechnet, dies ist etwa ein Drittel der gesamten Leistungen der obligatorischen Krankenpflegeversicherung (OKP). Hinzu 
kommen die Leistungen $\mathrm{zu}$ Lasten der weiteren Sozialversicherungen (Invaliden-, Unfall- und Militärversicherung), welche ebenfalls über den TARMED abgerechnet werden.

Zwar wird der überwiegende Teil der OKP-Leistungen im praxisambulanten Bereich erbracht (knapp 7 Mrd. Franken), jedoch hat sich der spitalambulante Bereich (4 Mrd. Franken) aufgrund der Verschiebung von stationären hin zu ambulanten Leistungen in den vergangenen Jahren relativ dynamisch entwickelt. Der ärztliche ambulante Tarif wird damit für den gesamten OKP-Bereich immer wichtiger und der Reformbedarf drängender. Denn: Fehltarifierungen hemmen technologische Entwicklungen, befeuern Kostensteigerungen und können gesellschaftlichen und politischen Trends (wie «ambulant vor stationär») zuwiderlaufen.

Ein TARMED, der nicht mehr sachgerecht und wirtschaftlich ist, führt also im besten Fall zu einer Fehlallokation der immer knapper werdenden finanziellen Mittel der OKP und hat damit direkte volkswirtschaftliche Implikationen. Im schlechtesten Fall zementiert der TARMED gar verkrustete Strukturen und bringt eine Fehlversorgung im Schweizerischen Gesundheitssystem mit sich. Die Schweiz hat nach den USA, gemessen an der Wirtschaftsleistung, das zweitteuerste Gesundheitssystem der Welt. Es liegt auf der Hand, dass sich eine solche Wohlstandsgesellschaft aller wirksamen Mittel bedienen sollte, um das Gesundheitssystem effizient zu halten. Das Mittel dazu sind nicht zuletzt moderne und sachgerechte Tarife, die regelmässig weiterentwickelt werden.

\section{Was wird besser mit dem TARDOC?}

Was auf den ersten Blick wie eine Kopie des TARMED aussieht, offenbart sich beim genaueren Hinsehen als komplette Erneuerung. Der TARDOC bildet im Vergleich zum TARMED das heute relevante ambulante ärztliche Leistungsspektrum ab. Das umfasst nicht nur die Bereinigung von nicht ambulant erbrachten Tarifpositionen und die Aufnahme von neuen Verfahren und technischen Möglichkeiten, sondern beispielsweise auch die verbesserte Abbildung der hausärztlichen Tätigkeit und die Neustrukturierung von Kapiteln. In der Projektphase der Erarbeitung der Tarifstruktur sind die Anliegen der freipraktizierenden Ärztinnen und Ärzte und der Spitäler eingeflossen. Die Aktualität und verbesserte Sachgerechtigkeit des TARDOC schlägt sich zudem in den Parametern des Tarifierungsmodells nieder. Die Infrastruktur- und Personalkosten des TARDOC basieren auf aktuell verfügbaren Daten. Sofern keine verwertbaren Daten verfügbar waren, wurden notwendige Annahmen auf Basis des heutigen Wissensstands getroffen. Dass mit dem TARDOC eine tarifpartnerschaftliche Lösung in Bezug auf das aktualisierte Regelwerk und die Mengenund Zeitlimitationen präsentiert werden kann, wurde im Projektverlauf nicht immer für möglich gehalten. Dieser Erfolg ist dem grossen Willen zur Erreichung eines gemeinsam revidierten Arzttarifs und den dementsprechenden Anstrengungen der in den Verhandlungen involvierten Partner zuzuschreiben. Wie wichtig die Auseinandersetzung aus beiden Blickwinkeln, Leistungserbringer und Versicherer, für die Qualität der revidierten Tarifstruktur ist, zeigte sich in Präzisierungen, die sich im Gespräch und in den Verhandlungen ergaben und vorgenommen wurden. Dies geschah immer im Bewusstsein, dass eine Tarifstruktur nie fertig ist, sondern zwingend einer laufenden Weiterentwicklung unterworfen sein muss.

\section{TARDOC bildet im Vergleich zu TARMED das} heute relevante ambulante ärztliche Leistungsspektrum ab.

Deshalb besteht das beim Bundesrat eingereichte TARDOC-Lösungspaket aus der Tarifstruktur, welche von einer Tariforganisation sowie vereinbarten Revisionszielen und -prozessen flankiert wird. Diese weiteren wichtigen Elemente einer zukunftsgerichteten Tarifstruktur-Lösung wurden von curafutura, FMH und MTK bereits vereinbart und aufgegleist. Die Tariforganisation existiert seit 2015. Die ats-tms AG Geschäftsstelle ist seit 2016 operativ und leistete einen massgeblichen Beitrag an der gemeinsamen Entwicklungsarbeit mit den Tarifpartnern. Zurzeit werden vom Tarifbüro die notwendigen Vorkehrungen für eine Einführung des TARDOC per 2021 getroffen. Die ats-tms AG bietet sich damit auch als nationales Tarifbüro für den ärztlichen ambulanten Tarif an, welches der Bundesrat in seinem Massnahmenpaket zur Kostendämpfung vorsieht.

\section{Ein aktueller Einzelleistungstarif als Grundlage für weitere Entwicklungs- schritte}

Der TARDOC ist wie der TARMED eine Einzelleistungstarifstruktur. Heute wird eine Einzelleistungsstruktur im Tarifwesen immer mehr in Frage gestellt, da sie Anreize für eine Mengenausweitung schaffe. Es werden daher von verschiedener Seite Pauschalen gefordert, welche die Einzelleistungsstruktur ersetzen sollen. Aber jede Tarifstruktur, egal ob Einzelleistungstarif oder Pauschale, hat ihre spezifischen Anreize. 
Aus Sicht der beiden Tarifpartner ist das Vorhandensein eines aktualisierten und sachgerechten Einzelleistungstarifs, welcher die Kriterien der Wirksamkeit, Zweckmässigkeit und Wirtschaftlichkeit erfüllt, eine Voraussetzung für weitere Entwicklungsschritte. Mit der nun vorliegenden Tarifstruktur TARDOC können jährliche Anpassungen erfolgen, und die Einzelleistungsstruktur kann auch als Grundlage für die Bildung von Pauschalen in geeigneten Fachgebieten dienen. Solche Pauschalen müssen nicht unbedingt in der Tarifstruktur TARDOC realisiert werden, sondern können auch zwischen der Ärzteschaft und den Versicherern beispielsweise auf Basis des TARDOC individuell ausgehandelt werden. Pauschalen könnten dort eingesetzt werden, wo das Leistungspaket sinnvoll und klar abgegrenzt werden kann und der Zeitbedarf für die Leistungserbringung eine geringe Streuung aufweist (beispielsweise im Operations- oder Radiologiebereich). Die zusammengefassten Leistungen sollten also über ein Patientenkollektiv gesehen in etwa gleich lang dauern.

\section{Ein Pauschaltarif kann den Einzelleistungstarif nie komplett ablösen oder ersetzen.}

In der derzeitigen kontroversen gesundheitspolitischen Diskussion scheint der Pauschaltarif plötzlich die Lösung für alle Probleme zu sein. Man muss sich jedoch bewusst sein, dass ein Pauschaltarif nie den Einzelleistungstarif komplett ablösen oder ersetzen kann, da es immer auch kombinierte Krankheitsbilder, Untersuchungen und Behandlungen geben wird, die sich nicht sachgerecht in Pauschalen abbilden lassen. Zudem kann ein Pauschaltarif bei schlechter Differenzierung Anreize zur Risikoselektion aufseiten der Leistungserbringer schaffen.

Wie bereits im Artikel von Ende August 2019 in der Schweizerischen Ärztezeitung erwähnt, sieht die Krankenversicherungsverordnung vor, dass eine neue Tarifstruktur keine Mehrkosten verursachen darf. Deshalb bekennen sich beide Tarifpartner zur Kostenneutralität. curafutura und FMH haben dem Bundesrat jeweils ein eigenes Konzept zur Sicherstellung der Kostenneutralität, das heisst der kostenneutralen Überführung vom alten in den neuen Tarif, eingereicht. Während curafutura eine Normierung (lineare Absenkung) der Taxpunkte in der Tarifstruktur vorsieht, möchte die
FMH die Kostenneutralität über die Anpassung der Taxpunktwerte steuern und die betriebswirtschaftlich gerechnete Tarifstruktur belassen. Wichtig zu erwähnen ist aber, dass beide Tarifpartner die exakt gleiche Tarifstruktur beim Bundesrat eingereicht haben. Damit ist es dem Bundesrat überlassen, welches der beiden Kostenneutralitätskonzepte er zur Anwendung bringt.

\section{Ausblick}

Die Ablösung des TARMED durch eine revidierte zeitgemässe Tarifstruktur, die über ihre institutionalisierten Organe und Prozesse die fortlaufende Weiterentwicklung gewährleistet, ist dringlich. Mit dem TARDOC ist eine Lösung am Start, die die Abrechnung aller ambulanten ärztlichen Leistungen auf eine neue, zukunftsweisende Basis stellt. Aus Sicht der Tarifpartner curafutura und FMH ist es jetzt am Bundesrat, so rasch als möglich den Startschuss zu geben, damit der TARDOC 1.0 in der OKP eingeführt und der kontinuierliche Entwicklungsprozess, mit dem TARDOC als lernendes System, gestartet werden kann. Nur so wird sichergestellt, dass die Aktualität der ärztlichen Praxis und die korrekte Abrechenbarkeit zukünftig im Einklang stehen und im Schweizer Gesundheitswesen eine nachhaltig sachgerechte nationale Arzttarifstruktur-Lösung verankert ist.

\section{Eckwerte des TARDOC}

- Einzelleistungstarifstruktur zur Abrechnung ambulanter ärztlicher Leistungen in der Arztpraxis, in ambulanten ärztlichen Institutionen und im Spital

- 71 Hauptkapitel mit Unterkapiteln

- 2700 Tarifpositionen (Zeit- und Handlungsleistungen)

- Anwendungs- und Abrechnungsregeln auf Ebene Tarifstruktur, Kapitel sowie Tarifposition

- Tariforganisation ats-tms AG (www.ats-tms.ch), gegründet 2016; aktuelle Trägerschaft: curafutura, FMH und MTK

- Einreichung beim Bundesrat durch curafutura und FMH am 12. Juli 2019 